\title{
How benchmarking promotes farmer and veterinarian cooperation to improve calf welfare
}

\author{
C. L. Sumner, (i) M. A. G. von Keyserlingk, (1) and D. M. Weary* (1) \\ Animal Welfare Program, Faculty of Land and Food Systems, University of British Columbia, Vancouver, BC V6T 1Z6, Canada
}

\begin{abstract}
Little is known about the combination of factors that motivate changes in calf management on dairy farms. Providing information to farmers may help promote change, but it is unclear how this approach affects and is affected by the farmer's relationship with the advisors such as the herd veterinarian. The goal of this study was to understand how benchmarking measures related to calf immune development and growth affected farmer and veterinarian cooperation and influenced the farmer's view of the veterinarian as an advisor for calf management. Veterinarians provided their clients $(\mathrm{n}=18$ dairy farms in the lower Fraser Valley of British Columbia) with 2 benchmark reports providing information on transfer of passive immunity and calf growth. Farmers were interviewed before and after receiving these reports to understand how they perceived their veterinarian as a calf advisor. Qualitative analysis identified 2 major themes indicating that benchmarking (1) improved farmer perception of their veterinarian's capacities to advise on calves and (2) strengthened the social influence of the veterinarian. We conclude that benchmarking can help promote stronger relationships between farmers and veterinarians.
\end{abstract}

Key words: animal welfare, extension, theory of planned behavior, attitude

\section{INTRODUCTION}

Dairy calves are at increased risk of morbidity and mortality when colostrum management is inadequate (Windeyer et al., 2014). Calves are also at risk of poor growth when provided lower rations of milk before weaning (Khan et al., 2011). The dairy farmer is the primary care provider for the calf, so it is logical that farmers are the focus of efforts to motivate changes (i.e., adoption of management practices) that address

Received January 19, 2019.

Accepted August 25, 2019.

*Corresponding author: dan.weary@ubc.ca these and other issues. In a recent study, our group provided benchmarking reports with information on how well preweaned calves acquired passive immunity from colostrum and how well calves were growing (Atkinson et al., 2017). After receiving these reports, the majority of the farmers made changes to their calf management (e.g., increased milk allowance or increased amount of colostrum at first feeding) that led to improvements in these outcomes (i.e., ADG or improved passive transfer of immunity; Atkinson et al., 2017). These farmers were also interviewed to determine how access to the information provided in the benchmarking reports motivated changes in management (Sumner et al., 2018).

Providing information is one way to promote behavior change, but behavior is also influenced by other factors, including the expectations of other people who influence decision making (Bicchieri, 2017). This influence of others is often referred to as a subjective norm (i.e., when a person engages in a behavior they believe they are expected to perform; Ajzen, 1991). Some recent work has described the concept of social influence (the influence of external social factors on an individual's thoughts, feelings, and behavior; Turner in Swinkels et al., 2015) on farmer decision making. For example, Ellis-Iversen et al. (2010) found that farmers were more likely to make changes in management related to disease when motivated by a trusted advisor.

The theory of planned behavior postulates that in addition to a person's attitude and perceived behavioral control toward a phenomenon, subjective norms influence a person's intention to act (Ajzen, 1991). In other words, people whose perspectives matter to us influence how we believe we should behave. Dairy farmers consider their veterinarian an influential advisor on topics related to animal welfare (Kauppinen et al., 2010; Pothmann et al., 2014; Wolf et al., 2016). For example, the veterinarian is considered an important influencer of social norms related to mastitis control (Jansen et al., 2010; Swinkels et al., 2015). On many farms, the adult lactating cow is the focus of systematic data collection (e.g., milk yield, fertility) and the primary focus of the dairy veterinarian (LeBlanc et al., 2006). It is not clear to what extent farmers consider 
their veterinarian an important influence on decisions about calf management, in particular decisions about colostrum and milk feeding.

In our benchmarking study (Atkinson et al., 2017; Sumner et al., 2018), the veterinarian played a central role in providing the benchmark reports to the farmers. Therefore, we focused on the veterinarian as a reinforcer of subjective norms for the current study. The goal of the current study was to understand how dairy farmer and veterinarian cooperation during a calf benchmarking study influenced farmer perspectives of the veterinarian as an advisor for calf management.

\section{MATERIALS AND METHODS}

This study was approved by the University of British Columbia Behavioral Research Ethics Board under no. H14-03196. All participants provided written consent.

\section{Study Design}

We were interested in understanding how benchmarking calf immune system development and growth with their veterinarian influenced the farmer's perspective about their herd veterinarian as an advisor in calf management. This emphasis on context and understanding meaning are consistent with a critical realist approach (Maxwell, 2012).

The research team for the benchmarking study comprised all 3 authors and a fellow graduate and undergraduate student. For the current study, the first author conducted all interviews. The first author was a PhD student with experience interviewing farmers and had a background in animal management with research interests in engaging people directly responsible for animal care to improve management practices. We assumed that the farmer would be the best choice to engage for improving calf management practices and that provision of information, coupled with a meeting with their veterinarian, would lead to improved management. We also assumed that the veterinarian would be the most relevant farm advisor to approach the farmer and advise them on how they could interpret benchmarking reports and make any relevant changes in management. The University of British Columbia has a long history of working with the local dairy community, and as such the research team had a previous professional relationship with the participants. We explained to all participants that our intention was to assess how benchmarking calf immune system development and growth could inform changes in farm management.

The participants in this study were the same as those described in Sumner et al. (2018). Details of the sampling rationale and recruitment process are provided in
Sumner et al. (2018). Briefly, we recruited a convenience sample of 18 commercial dairy farms in the lower Fraser Valley of British Columbia and interviewed farm staff involved in calf management, including owners, herd managers, and calf managers. This sample provided a diverse range of people who provided direct care to the calves. For the purpose of this study, these participants are collectively referred to as "farmers." Participants in this study were recruited through their herd veterinarian. The researchers first approached the veterinary clinic to determine their interest in the study. Once veterinarians agreed to assist with the study, they approached clients that fit the inclusion criteria for the biological data collection (e.g., herd size and reliance on dam colostrum; see Atkinson et al., 2017) to determine their interest in participating in the study. If interested, an initial in-person meeting was scheduled between the farmer, veterinarian, and researchers. Farmers were provided with a description of the study and consent forms and given time to decide whether they wanted to participate. In-person meetings were scheduled on 19 farms and, a total of 21 farmers on 18 of these farms agreed to participate in the study (1 person declined for reasons unknown).

During the study, each farmer received 2 reports separated by 10 wk. These reports described serum total protein from individual calf blood samples, ADG (as estimated from heart-girth tapings), and information on management practices used on the farms (for a summary of biological findings, see Atkinson et al., 2017). In addition to the farm's own data and how the data compared with that from other farms, relevant information was discussed at each meeting (e.g., the effects of increasing milk ration on calf growth), and, when needed, props (e.g., a colostrometer for testing colostrum quality) were used to facilitate the discussion. Veterinarians presented both benchmarking reports to their clients. Veterinarians were encouraged to tailor each discussion around the needs of each individual client. Researchers from the University of British Columbia participated in the meetings to provide support to veterinarians on report content or relevant supporting information. The first author attended all benchmarking report meetings. For the first meeting, 3 other members of the research team were also present; the first author was the only member present at the second benchmarking report meeting. A version of these reports is provided in Supplemental File S1 (https://doi.org/10.3168/jds.2019-16338).

\section{Interview Guide, Data Collection, and Participants}

We used the theory of planned behavior to develop semistructured interview questions based on the theo- 
retical construct of subjective norms. Subjective norms are the perceived social expectations to perform a behavior (Ajzen, 1991). We specifically focused on the veterinarian as a reinforcer of subjective norms because they delivered the reports to the farmers. Additionally, the extent of involvement that veterinarians had in calf management in this study was novel; therefore, we wanted to learn how this interaction changed the farmer's perception of the veterinarian reinforcing a subjective norm for calf management. Based on the subjective norms, we asked the following research question: How does veterinarian involvement with these reports change how farmers think about managing their calves?

The first author solely conducted all interviews with farmers before and after $(\sim 34 \mathrm{wk}$ apart) farmers received their benchmark reports from their veterinarian (see also Sumner et al., 2018). The first round of interviews was conducted from April to June 2015 and were approximately $45 \mathrm{~min}$ in length. The second round of interviews were conducted September to October 2015 and were approximately $32 \mathrm{~min}$ in length. Most interview questions were first tested in the pilot study, but some questions were not pilot tested and thus were refined during the study. Before farmers discussed their benchmark reports with their veterinarian, they were asked whether their veterinarian influenced their calf management. Probing questions included the following: (1) Do you approach your veterinarian to talk about calves? (2) Does your veterinarian approach you to talk about calves? and (3) Do you ask the veterinarian about your calves' health? After farmers discussed both benchmarking reports with their veterinarian, farmers were asked whether the veterinarian's involvement in the benchmarking influenced the way they managed their calves. Probing questions included the following: (1) Were there changes in the amount you discussed calves with your veterinarian? and (2) Did you find these discussions beneficial? All interviews were audiotaped and conducted by the first author on the participants' own farms.

\section{Data Analyses}

Audio files were transcribed by a professional service, and transcripts were compared with the original files to ensure fidelity. We used QSR's Nvivo (version 10.2.2; https://www.qsrinternational.com/) for analysis. We analyzed the data in 2 separate rounds of coding. As described in Sumner et al. (2018), for the first round of coding we used constructs from the theory of planned behavior (i.e., attitudes, perceived behavioral control, and subjective norms) for code labels. For the current study, our focus was on the relationship between the farmer and the veterinarian, and we only analyzed data coded as subjective norms related to veterinarians (data coded as subjective norms related to farmers, family, and farm staff were the focus of Sumner et al., 2018). For the second round of coding, we focused on coding patterns that emerged from the first round. The lead author and another trained individual helped to develop a list of code definitions related to subjective norms that were then used to code a subset of transcripts (chosen randomly) from before and after the benchmark reports; discrepancies on how data were coded were discussed until consensus was reached. The lead author then coded the remaining interviews.

In the subsequent step of coding, the data coded as the subjective norms related to the veterinarian were then further coded using applied thematic analysis (see Guest et al., 2014). During this step, emergent themes and initial codes were first described after a read-through of the data and organized into a codebook (provided in Supplemental File S2: https://doi.org/10 .3168/jds.2019-16338) based on themes and related code labels and definitions. Another trained individual coded a subset of the data (chosen randomly) using the codebook. The lead author and this individual met to discuss how the data were coded and resolve discrepancies. Modifications to the codebook were included in the final version based on this meeting.

Saturation was achieved at multiple points in the data collection and analysis (see Saunders et al., 2018). Data saturation was achieved during collection, in part through repeat interviews where farmers were asked questions and follow-up questions on the same topic. Additionally, farmers were asked to discuss any information we had not covered in the interviews. This provided confidence that data collection was exhaustive for each participant in that we finished interviews when no new information arose during these discussions. Saturation of themes was achieved through the coding process when no new themes or subthemes were interpreted in the data analyses. Coding had begun during the first round of interviews and continued after the second round of interviews were complete; thus, theme saturation was contingent on an iterative process during and after data collection.

Quotes were selected to represent examples of a given category within each theme; we specifically identified statements reflective of many responses and that more clearly expressed a given concept. Quotes have been modified for length and clarity; ellipses indicate where text was omitted to reduce quote length, and parentheses indicate the authors' addition to the text in cases where clarification was warranted. Participant numbers assigned during data collection have been altered in this article to protect anonymity. 


\section{RESULTS}

We present 2 major themes that emerged from the analysis of participant responses indicating how benchmarking influenced dairy farmers' perceptions about their veterinarian as an advisor for calf management. First, benchmarking provided an opportunity for veterinarians to demonstrate their expertise on topics related to calf management. Second, benchmarking increased interaction between farmers and veterinarians on issues of calf management. Both themes include confirming and disconfirming views within the topic to represent the diversity of farmer perspectives. The themes are organized chronologically, starting with farmer perspectives on their relationship with their veterinarian before benchmarking and then describing changes in these perspectives after benchmarking.

\section{Theme 1: Perception of the Veterinarian as an Advisor}

Before receiving the benchmark reports, farmers saw their veterinarian as a source of information and training, with expertise to provide guidance on topics related to disease and pain management. After meeting with their veterinarians to discuss the reports, veterinarians were further recognized for their ability to address other issues related to calf management, including milk allowance and colostrum management.

Before Benchmark Reports. Farmers described their veterinarians as educators on topics of calf management, including taking an active role in educating farmers about new calf management techniques. For example, Farmer 16 reported, ". . . the clinic that I'm with are very proactive in educating people." Veterinarians were described as teaching farmers through different means such as in-clinic seminars or through use of protocols and procedures for dehorning, colostrum management, or treating health-related problems such as diarrhea and dehydration. Veterinarians were also described as training new farm staff on using protocols for calves:

... When somebody starts out, (the veterinarian) explains what the protocol is .... When I first started (taking care of calves), we talked about it a lot, and (the veterinarian) showed me different things, how to do this and that. (Farmer 4)

Veterinarian expertise on topics of calf management emerged as a feature of how farmers described their veterinarian's capacity to advise on calf management. Veterinarian expertise was considered an extension of their background because "(they) go to school for that many years" (Farmer 13), "they have a little more science backing them" (Farmer 11), and "because (they) see more research than I do" (Farmer 6). Farmers considered their veterinarian a source of information on health concerns such as respiratory disease, diarrhea, and managing pain during dehorning. Some farmers considered their veterinarian a source of information on topics of nutrition (i.e., feeding grain, hay, and water), calf growth, and weaning.

Farmers used their veterinarian's knowledge of calf management to confirm opinions they had encountered from other sources:

... (With treating calves) . . . I'll do my own research, but I'll also ask them, have their opinion. ... . They're the ones that I would turn to if I'm having an issue with certain things that I couldn't figure out. (Farmer 13)

Veterinarian expertise was seen as more reliable than other sources of available information from other people (i.e., nutritionist or other farmers) or from print and online sources. As Farmer 14 explained, when making changes to calf management, he relied on expertise ". . . mainly (from) ... the vet, not so much other people." Placing value in learning from veterinarians was based on farmers valuing multiple opinions and views about calf management: ". . . They know what they're talking about, and it's just another idea and opinion pooled in with ours . .." (Farmer 13). However, some farmers did not view their veterinarian as having expertise on calves, thus undermining the perceived usefulness of veterinarian advice: "He has some influence, but he isn't a calf specialist" (Farmer 9).

Farmers trusted their veterinarian as an advisor for calf management because the veterinarian was already established as a trusted advisor on the lactating herd. This established role on the farm, coupled with a familiarity with the farm's overall operations, engendered a sense of trust. As Farmer 3 described, the benefit of having the veterinarian involved in calf management was that ". . . he understands what I'm doing here."

Trust was also associated with the veterinarian's position of linking farmers with other farmers, thereby bringing relevant knowledge from other farms. The veterinarian's familiarity with other farms was seen as an asset, and farmers described this link with other farmers as beneficial for their calf management because the veterinarians ". . . see more farms, more varieties, and they see a little better what works and what doesn't work. . . ." (Farmer 15). Sharing ideas about other farms was seen as useful in thinking through ways 
to address problems: ". . . If you have a problem, (the veterinarian) might start to say, 'Well, these are some other problems that other (farmers) have,' . . . and you can sort of help each other ..." (Farmer 7). Farmers placed value on their veterinarians as a social link because it helped promote new ways of managing the farm:

I like to listen to what (other farmers) are doing and see why they do it the way they do it. So, that's why it's nice to have other people, like the vet, coming and saying this is what (other farmers) are doing. (Farmer 11)

Veterinarians also promoted farmers meeting each other to discuss calf-rearing practices, including arranging introductions and farm visits:

... (My veterinarian) has in (their) head a great calf-rearing system. ... And if (they) know the other farmer (with this system), . . (they) would have no problem offering me (their phone number) - and there are some farms (they) say, "Go talk to so and so, and go have a look at their facility." (Farmer 16)

After Benchmark Reports. After meeting to discuss the benchmark reports, farmers noted that their veterinarian displayed knowledge on calf topics that they had not previously discussed. Farmer 2 explained that although they had discussed calf health issues with their veterinarian, colostrum and milk allowance had never been mentioned: ". . . It was new for us to discuss (colostrum and milk allowance)." Weight gain was also a new topic for farmers and veterinarians. The benchmark study monitored calf growth during the study and provided an opportunity for the veterinarian to contextualize this performance:

... To be honest, I wouldn't even know what to say what an average daily gain would be for a calf. ... I was the highest but, I mean, other than that, I couldn't tell you is it good or is it bad. How does it compare with other studies? .. . Well, the vet gave some answers in that aspect. (Farmer 7)

Additionally, after the benchmark, farmers described their veterinarian's role in developing protocols for topics such as nutrition: ". . . We work with (our veterinarian) . . . in developing more of a protocol and being more consistent in our approach to feeding our calves" (Farmer 2).
Having advice on topics related to the benchmark reports was noted as valuable because of perceived veterinarian expertise: "I think they were crucial conversations to have and involve him ..." (Farmer 16). Veterinarian expertise was noted as contributing to farmers' ongoing efforts to identify and solve problems. Veterinarians confirmed ongoing concerns farmers had about their calves that were directly related to the benchmark study, such as improving milk feeding to promote gains in calf weight: “. . . There's room to improve on the daily gains. ... (My veterinarian) and I have talked about that because that really bugs me. I want to get that up" (Farmer 12). Veterinarians also confirmed farmers' concerns for calf management practices not directly addressed in the benchmark study. Contrasting opinions were also noted regarding veterinarians' expertise on issues such as feeding hay to preweaned calves:

... I had heard that hay was actually detrimental to growth because it took up room in their rumen when it wasn't necessarily ready to go. I mean, you talk about disagreeing . . . when we were talking with (our veterinarian). I disagree with putting hay in front of them at any kind of amount any earlier than what we're doing. . . . (Farmer 18)

Some farmers reported no change in how they viewed their veterinarian's capacity to advise on calf management after the reports. Some, such as Farmer 13, still recognized the veterinarian's perspective, but the benchmark did not promote a change in their perception of their veterinarian's role in decisions regarding calves: "I think it's the same, to be honest. . . . I think he has a lot of valid opinions and everything. . . . I mean, it's nice having an extra voice. . . . But it wasn't like his opinion was the deciding factor." Another reason for a lack of change was because farmers already considered their veterinarian's expertise when making decisions about calves. Farmer 16 explained that they anticipated their good performance on the colostrum management because they relied on their veterinarian's expertise: "Being (near) the top, I kind of had in my head we'd be there somewhere. . . . I'm very in tuned with what (my veterinary clinic) is up to with calves."

\section{Theme 2: Benchmarking Strengthened Veterinarian Social Influence in Calf Management}

Benchmarking provided farmers and their veterinarians space to discuss new topics and adopt new practices related to calf management. Before farmers received their benchmark reports, the farmer-veterinarian rela- 
tionship regarding calf management was based primarily on reacting to health-related problems. Most farmers reported they had a cooperative relationship with their veterinarian that was focused on solving problems with calf management and sometimes consulting on future planning for calf management. After discussing the benchmark reports, farmers described changes in the relationship related to feeling motivated to improve calf management and increased interaction with their veterinarians.

Before Benchmark Reports. Before receiving the benchmark, many farmers indicated that they worked together with their veterinarian to solve problems related to calf management. For example, Farmer 15 described a mutual interest in calves:

If we have any questions, we'll talk to them. If they see something that can be improved, or something comes through a study or whatever and that's of interest, they'll let us know.

However, when to act rested on perceptions of what was considered a problem, usually related to the health of the calves: ". . . I don't think in calf management they're usually involved unless there's a whole bunch of calves dying" (Farmer 10). In addition to addressing current problems with calves, some farmers turned to their veterinarian to seek advice on future decision making about calves. A common feature of this relationship was that farmers sought their veterinarian's advice on plans to improve calf housing, as Farmer 15 describes: "We talked to (our veterinarian) a little while ago about (building) a (calf) barn."

Veterinarians approached their clients mainly about health-related topics such as vaccination protocols and diagnostics, keeping records for birth, mortality, and colostrum management. Veterinarians also played a role in encouraging the uptake of management practices that farmers viewed as improving calf welfare. Farmer 18 described the benefits for the calf and himself of using pain relief during dehorning:

. . To take the pain out of the picture is huge, if you care at all about the animal. Otherwise it's just, you know, barbaric. But that's the way things have been done for a long time. And people have to change that mindset.

In addition to concerns for welfare, following veterinarian advice was linked in some cases to constraints such as time or financial cost. A deciding factor in determining whether the advice was followed by some farmers was whether it was perceived as practical:
... If they give me an idea that's easy enough to do and might help, then yeah. But if they give me an idea that I'm going to end up spending four hours a day with my calves, well, no. (Farmer 5)

For others, following veterinarian advice for calf management was linked with the veterinarian's ability to improve the productivity of the herd: ". . . (He'll) just tell me what's going to make the farm better in the end, make us more money ... " (Farmer 12). Deciding to follow the veterinarian's advice for managing calves was also based on the status of calves compared with other aspects of the farm. Some farmers may have considered calves a lower priority on the farm. For example, Farmer 16 suggested that this was why some farmers did not use pain relief during dehorning: ". . . It's hard to get people to adopt humane things. . . . Dehorning with painkillers and putting the calf to sleep, that investment is only humane."

Farmers varied in the extent that they contacted the veterinarian regarding calf health problems. Some farmers reported not calling their veterinarian for sick calves, whereas others reported calling only when major disease-related outbreaks occurred. Some farmers acknowledged that the veterinarian rarely visited the farm solely for calves but would still discuss calf issues during routine visits for the lactating cows: "Oh, you know, our vet will look at our calves maybe a couple of times a year in reality. . . . I do the herd health. So, (the veterinarian and I) talk about everything during that time" (Farmer 18). Relying on routine visits for the lactating herd to discuss calves reinforced the limited interaction between farmers and veterinarians for addressing nonroutine calf problems. For at least 1 farmer, the exclusion of calves from routine herd health visits was problematic:

First, I have to throw it by (the owner), because (he) doesn't want the vet time to be spent with calves. (Imitating the owner): '(Veterinarians) do the herd health, you know?' But a calf is herd health too, in my opinion. (Farmer 1)

Some farmers thought that the veterinarian should have a more active role in calf management, identifying themselves as being responsible for not involving their veterinarian in calf management. For example, Farmer 3 stated that the limited veterinarian involvement was ". . . probably my fault for not involving him."

Farmers described the relationship with their veterinarian as involving shared accountability. For example, some farmers acknowledged that the veterinarian 
helped ensure accountability to meet industry requirements for using pain relief during dehorning:

Yeah, our veterinarian was the one who tried to get us to (use pain relief during dehorning). And he said, 'You guys have to do this because we're going to have to do it eventually, like, legally. So, you guys are going to have to do it now.' (Farmer 11)

Farmers also were keenly aware of the negative sanctions, such as disapproval, from other groups such as the public. For example, they felt that pain relief for dehorning was an important management practice and acknowledged their veterinarians' influence in meeting public expectations:

I think a bit of it is a snowball effect, you know. You take (pain relief for) dehorning . . . it was just a little ball. You know, half a dozen farmers did it. It was just a little ball. But vets always had to push it, they were pushing it, pushing it, pushing it. But once that ball got to be big enough, it kind of just started rolling on its own. And through other farmers talking about, to vets maybe talking about it, to reading about it, to hearing comments from people outside of the industry saying how they don't like (the lack of pain treatment), you just kind of get hit from all areas of your senses, and areas of your social, and the areas of your peers. That speeds that snowball effect. (Farmer 16)

Involving the veterinarian in calf management was also seen as encouraging negative feedback from veterinarians and a reason why some farmers may have refrained from seeking their advice for calf management: "I think it's more to do with the farmers not . . . wanting to change . . . if he's going to get mad at you for your calf management" (Farmer 10). In contrast, veterinarian praise was considered a positive outcome of veterinarians taking a more active role in calf management, as described by 2 farmers on Farm 10:

Participant 2: . . When you have a vet . . wandering around (and saying), 'Oh, your calves look really good.'

Participant 1: He encourages you, right?

Participant 2: That probably means that you're better than average, right? . . . You get the tone of what he's saying. . . .
After Benchmark Reports. After receiving their benchmark reports, farmers that described changes in how they interacted with their veterinarian described the value of a more interactive relationship. The most common change in the farmer-veterinarian relationship was increased discussion about calf management. This was valued during routine herd visits:

If we're having issues or we think something's not right, I don't have a problem spending (more time) - or even if it's just, 'How are your calves? Oh, let's go look at them,' right, and then get a bit of a visual, talk about them for five minutes. That's fine. (Farmer 8)

Talking to veterinarians about calves motivated farmers to improve management practices. Farmer 7 described the influence of talking to the veterinarian on his willingness to make changes in calf management:

Any time you talk about things you sort of get motivated to do it even better, you become more conscious of it, you know, you pick up stuff. . . . I think you become more conscious of doing good colostrum management. I mean feeding more milk, or a denser ration, to a younger calf.

Farmers indicated that discussion with their veterinarians about the benchmark reports improved their understanding of the information: "Now, if (the reports are) actually handed to you and explained to you . . . then you actually absorb it. You think about it" (Farmer 13). In addition to increased discussion about calves, farmers reported other interactions indicating that the veterinarian's more active role in calf management helped improve calf management. Farmer 15 described how their veterinarian approached them with new products such as using larger bottles for colostrum and daily milk allowance:

... We're giving the newborn calves a lot more colostrum right off the bat, the biggest bottles you can buy. . . . I couldn't really find anything that would at least fit our hutches (for feeding more milk). . . . But then (our veterinarian) all of a sudden out of the blue found these bottles.

The benchmark reports prompted increased interactions between veterinarians and farmers through use of ongoing diagnostics to determine the quality of colostrum that was fed to calves. Farmer 7 described how this testing motivated him to continue to improve managing colostrum: ". . . We did a bunch of colostrum 
(quality) tests. ... A lot of them failed. . . . Those are some things you sort of question. . . . It would be interesting to look into that, and I would delve into that more. ..."

Farmers valued their veterinarian being more aware of the problems on their farm, with specific reference made to the calves:

It was helpful to have (the veterinarian) here. I'm glad they were involved. . . . And it's good for them to know if I'm not doing a great job. . . . They can help me with that. (Farmer 3)

Additionally, farmers noted that the veterinarian's appraisal of their calves during the benchmark study affected morale on the farm:

(The veterinarian will) just go walking by (and say), 'They've grown,' . . . and those kids (who feed the calves) hear that . . . feedback, they're like, 'Oh wow, like so what we're doing obviously is helping,' and I think that's just kind of-it's now rewarding for them too, in a sense. (Farmer 4)

When farmers reported no change in their interactions with their veterinarians regarding calf management after benchmarking, it centered on reasons of economics: "I don't know if I want to pay him for it. That's the downside of getting a vet to do all these things" (Farmer 8). Concerns about personal autonomy in decision making also prevented changes in the farmer-veterinarian relationship: "I'm not influenced . . . I still know what's best for our farm." (Farmer 13). Additionally, farmers that already had a comprehensive relationship before the benchmark study reported that there was no change in the relationship after the benchmark. As Farmer 12 explained about the extent to which his veterinarian was involved in calf management, ". . . that's never changed. . . . (The veterinarian) develops our vaccine protocols, everything. So, he keeps an eye on the calves, makes sure that's all working. . .."

\section{DISCUSSION}

Our results indicate that involvement in benchmarking can enhance the perceived value of veterinarians as advisors in calf management and promote interactions between farmers and veterinarians on calf management. The theory of planned behavior was used to develop the interview guide and with the first round of coding during analysis. The data for the current study were collected with the expectation that discussing informa- tion about their calves with the veterinarian would influence how farmers viewed this relationship, including their normative beliefs about the veterinarian as a motivator for changing calf management. We assumed that the theory of planned behavior could provide guidance on understanding farmer motivation to improve calf management, in part because the veterinarian can reinforce subjective norms. We found that farmers' stories about their relationship with their veterinarians did not necessarily include a subjective norm interpretation (e.g., pressure to conform to their advice; see Rimal and Real, 2003); much of the discussion was on describing the farmer's perspective of the capacities of, and interactions with, their veterinarian. The following section discusses both the descriptive and normative influence of the herd veterinarian on calf management.

\section{Increased Farmer Perceptions of Their Veterinarian's Capacity to Advise on Calf Management}

Based on farmers' experiences with their veterinarians, benchmarking calf growth and colostrum management played a key role in increasing the veterinarian's value and influence as an advisor for calf management, in part because it increased the perceived capacity of the veterinarian to advise. Recognition of capacity has been identified as a benefit for participants in health interventions (Wathen and MacMillan, 2018). Additionally, Triste et al. (2018) identified the importance of technical advisors in dairy farming communities. In our study, farmer recognition of the influence of veterinarians appears to relate to their perceived capability to advise on calf management. Cialdini and Goldstein (2004) described this type of social influence as relying on soft tactics that are internal to the person rather than hard tactics that rely on social structures to enforce compliance.

The recognition by farmers that the veterinarian plays a role in education is consistent with the description in Oreszczyn et al. (2010) of individuals who negotiate boundaries that distinguish a farming community. In our study, some farmers described a demarcation between the farms and the research community on issues related to calf management and the veterinarian's role in bridging these two communities. Farmers in our study valued their veterinarian as a source of information, particularly in terms of interpreting scientific material, confirming ideas from other sources, and providing alternative or contrasting views. The veterinarians thus served a role in helping farmers "staying up to date with a kaleidoscope of perspectives on farming practice" (Sligo and Massey, 2007, p. 76). 
The ability of an expert to exert social influence, and the acceptability of their information, relies on the recipient acknowledging that the person has expertise (French and Raven, 1959). Farmer recognition of the veterinarian's expertise on calf management varied, but the benchmark study provided an opportunity for veterinarians to demonstrate this knowledge. This is an important step given that the veterinarian's capacity to advise is reliant on the farmer's perception of their expertise and that in some cases veterinarians are perceived to lack expertise (e.g., regarding treating protocols for organic herds; Vaarst et al., 2003; Duval et al., 2017).

For farmers who saw their veterinarian as a trusted source of information on calves, the advice given was viewed to be more reliable than that provided by other farm advisors. One exception was on topics related to feeding; on this issue, farmers seemed to place greater weight on advice from their nutritionist. This example suggests that better cooperation between farm advisors (including veterinarians and nutritionists) could be helpful. The degree of trustworthiness of the advisor, and the information they share, can influence the extent that farmers seek out their advisor's support (Sligo and Massey, 2007). Benchmarking was not always effective in promoting veterinary influence. For some farmers in our study, despite increased engagement with their veterinarian on the topic of calf management, the veterinarian was still not viewed as influential. Future work on farmer perception of trustworthiness of their veterinarian may help explain why some interventions are more successful than others.

The benchmarking process increased the veterinarian's role as a social link between farmers, indicating that veterinarians can play a role in sharing ideas about calves and facilitating communication among farmers. Sumner et al. (2018) noted that farmers varied in how they described their relationship with other farmers regarding calf management; although farmers did not consider other farmers as influential in their own decision making, they were interested in how other farmers managed their calves. The results of the current study illustrate the veterinarian's role in linking farms and in sharing ideas; this insider knowledge of multiple farms was described as a valued capacity.

\section{Strengthened Veterinarian Social Influence in Calf Management}

Delivering the benchmark reports to their clients increased interactions between farmers and veterinarians, in some cases solidifying the current relationship and in others stimulating a more proactive approach to calf management. Before our study, work on how farmers viewed their veterinarian as a social influence on calf management was limited. Our study demonstrates that farmers consider the veterinarian a social influencer for calf management and that increasing communication can strengthen this influence.

Communication played a central role in increasing the social influence of the veterinarian. During the study, farmers and veterinarians increased their interaction by communicating about calves because of the benchmark reports. The current study showed that some farmers and veterinarians were already interacting about calf management. For these farmers, benchmarking did not change the relationship per se but did serve to introduce new topics of engagement, providing more reason to communicate. The process also provided additional breadth to the topics discussed, including topics that were unrelated to the benchmark. These results are consistent with Triste et al. (2018), indicating that farmers valued talking to their veterinarians about calf management. In particular, through the processes of communication, the veterinarian's interpretation of the reports added to the farmer's understanding of the information presented. These reports also generated discussion about other concerns not included in the benchmark.

Farmers valuing discussion about calves has been noted in other studies. Vasseur et al. (2010) noted that one challenge with the feasibility of an advisory tool on calf management was that the time needed to conduct on-farm visits was lengthened by farmers wanting to discuss issues. Increased discussion also has benefits beyond the immediate concern. Wathen and MacMillan (2018) argued that increased interaction is a basis for building and maintaining trusting partnerships.

Our study provides evidence that some calf management practices are likely social norms. In general, norms are behaviors that people within an identified group believe others should conform to (Bicchieri, 2017). Norms are contingent on their acceptance by the group and are used to hold one another accountable to shared values (Southwood and Eriksson, 2011). We suggest that farmers' understanding of how they are expected to care for calves may vary with the specific practice. Social norms were most clear when farmers described the perceived negative sanctions imposed by their veterinarian, other farmers, and the public about not using pain relief for dehorning, illustrating the expectation that they should relieve pain during this procedure.

Both positive and negative sanctions based on social norms indicate how a group thinks something should be done (Rimal and Lapinski, 2015; Bicchieri, 2017). Some 
evidence exists that veterinarians can have positive and negative social influences on dairy farmers for mastitis reduction (Swinkels et al., 2015). Our study found that veterinarians also had both positive and negative normative influences on calf management. For example, farmers reported wanting to avoid negative sanctions from their veterinarians such as criticism and appreciating positive sanctions such as praise for how they reared calves. Dehorning is an example where farmers acknowledged their veterinarian's help in changing farm practice linked with negative sanctions. Moral influence is a function of social distance; the closer the relationship, the stronger the influence (Haidt, 2001). It is thus to be expected that the veterinarian had a social influence.

Farmers are also concerned about public perceptions (de Rooij et al., 2010) and are open to influence from veterinarians on matters that relate to public concerns. The practices we benchmarked may be less subject to social norms - for example, that farmers believe that other farmers in their social network are feeding increased amounts of milk to calves and feel they are expected to do the same. If we consider this belief about milk allowance to be a social norm, then we would expect it to reflect that members of the dairy farming community place value on calves having increased milk allowance (for more about values and norms, see Southwood and Eriksson, 2011). Some veterinarians consider milk allowance as having an ethical dimension due to concerns about calf hunger (Sumner and von Keyserlingk, 2018), although the extent to which farmers share this value remains unknown. The suggestion that calf milk allowance is emerging as a social norm indicates that veterinarians should consider adopting approaches that encourage increased milk allowance for calves and promote farmer deliberation about this topic (see Bicchieri, 2017).

Although we found many examples of the veterinarian's social influence on calf management, some farmers did not consider their herd veterinarian in this manner. Burton (2004) argued that the lack of influence relates to farmers valuing independence. Simply asking about the influence of others may also promote assertions about independence (Burton, 2004). We found that farmers expressed value in being able to make their own decisions and that this autonomy was related to how they considered their veterinarian's influence. Autonomy in decision making has been noted when farmers described other farmers as having no influence on their decision making about calves (Sumner et al., 2018). This may be related to what some have argued is a "do it yourself" attitude toward decision making (Jansen et al., 2010). Some have argued that it is incumbent on the veterinarian to adjust their approach to clients so that they are recognized as a potential advisor (Bard et al., 2017; Duval et al., 2017), and others have argued that it is also the responsibility of the farmer to recognize their veterinarian as a trusted advisor (Vaarst et al., 2003). Our study illustrates how both parties can work together to improve calf management.

\section{Interpreting this Study}

We sampled participants with no intention of representing the views of all dairy farmers. As noted in our companion paper (Sumner et al., 2018), farmers in this study were willing to try new ways to manage calves, including spending more time with their veterinarians discussing calves, and this characteristic is an important contextual feature of this study. Additionally, farmers did not pay their veterinarians for the time and advice associated with this study. We acknowledge that respondents may have wished to project a favorable image but argue that our study design reduced this risk by repeat visits to farms and multiple interviews (Sumner et al., 2018).

Our rationale for using the theory of planned behavior was to explore the role of the veterinarian, as an interpreter of the information in the benchmark reports, in motivating farmers to improve calf management. Information helps us understand motivational factors. Ajzen (1991) describes "salient information" as beliefs that are "the prevailing determinants of a person's intentions or actions" (p. 189). Information also influences the formation and stability of attitudes (as reviewed by Glasman and Albarracín, 2006) and the interpretation of norms (Bicchieri, 2017). However, a limitation with using the theory of planned behavior is reliance on internally motivating factors and not taking into account the influence of external factors such as environmental and economic conditions and a person's mental and physical health (Sniehotta et al., 2014). Future work with dairy farmers and veterinarians should consider the influence of these external factors and how they constrain or enable improvements in farm management.

\section{CONCLUSIONS}

Benchmarking calf management practices related to growth and colostrum management positively influenced farmer perceptions of the veterinarian as an advisor on calf management and promoted interactions over calf management. Benchmarking can motivate farmers to consider their veterinarian a social referent for calf management. 


\section{ACKNOWLEDGMENTS}

We thank fellow Animal Welfare Program members, especially Beth Ventura for her help in planning the study, Dax Atkinson for his collaboration in data collection, and Katie Koralesky and Katie Mills for their help with the data analysis. We also thank Hannah Wittman (University of British Columbia, Vancouver, BC, Canada) and Mette Vaarst (Aarhus University, Aarhus, Denmark) for their comments and suggestions on a previous version of this paper. Funding for Christine Sumner was provided by the University of British Columbia's 4YF doctoral fellowship program. This research was made possible by the funding for the biological outcomes (described in Atkinson et al., 2017) from Natural Sciences and Engineering Research Council of Canada's (Ottawa, ON, Canada) Industrial Research Chair program, with industry contributions from the Dairy Farmers of Canada (Ottawa, ON, Canada), British Columbia Dairy Association (Burnaby, BC, Canada), Westgen Endowment Fund (Milner, BC, Canada), Intervet Canada Corporation (Kirkland, QC, Canada), Novus International Inc. (Oakville, ON, Canada), Zoetis (Kirkland, QC, Canada), BC Cattle Industry Development Fund (Kamloops, BC, Canada), Alberta Milk (Edmonton, AB, Canada), Valacta (St. Anne-de-Bellevue, QC, Canada), and CanWest DHI (Guelph, ON, Canada).

\section{REFERENCES}

Ajzen, I. 1991. The theory of planned behavior. Organ. Behav. Hum. Decis. Process. 50:179-211.

Atkinson, D. J., M. A. G. von Keyserlingk, and D. M. Weary. 2017. Benchmarking passive transfer of immunity and growth in dairy calves. J. Dairy Sci. 100:3773-3782.

Bard, A. M., D. C. J. Main, A. M. Haase, H. R. Whay, E. J. Roe, and K. K. Reyher. 2017. The future of veterinary communication: Partnership or persuasion? A qualitative investigation of veterinary communication in the pursuit of client behaviour change. PLoS One 12:e0171380.

Bicchieri, C. 2017. Norms in the Wild: How to Diagnose, Measure, and Change Social Norms. Oxford University Press, Oxford, UK.

Burton, R. J. F. 2004. Reconceptualising the "behavioural approach" in agricultural studies: A socio-psychological perspective. J. Rural Stud. 20:359-371.

Cialdini, R. B., and N. J. Goldstein. 2004. Social influence: Compliance and conformity. Annu. Rev. Psychol. 55:591-621.

de Rooij, S. J. G., C. C. de Lauwere, and J. D. van der Ploeg. 2010. Entrapped in group solidarity? Animal welfare, the ethical positions of farmers and the difficult search for alternatives. J. Environ. Policy Plann. 12:341-361.

Duval, J. E., N. Bareille, C. Fourichon, A. Madouasse, and M. Vaarst. 2017. How can veterinarians be interesting partners for organic dairy farmers? French farmers' point of views. Prev. Vet. Med. 146:16-26.

Ellis-Iversen, J., A. J. Cook, E. Watson, M. Nielen, L. Larkin, M. Wooldridge, and H. Hogeveen. 2010. Perceptions, circumstances and motivators that influence implementation of zoonotic control programs on cattle farms. Prev. Vet. Med. 93:276-285.
French, J. R. P., and B. Raven. 1959. The bases of social power. Pages 259-269 in Studies in Social Power. D. Cartwright, ed. Institute for Social Research, Ann Arbor, MI.

Glasman, L. R., and D. Albarracín. 2006. Forming attitudes that predict future behavior: A meta-analysis of the attitude-behavior relation. Psychol. Bull. 132:778-822.

Guest, G., K. M. MacQueen, and E. E. Namey. 2014. Applied Thematic Analysis. Sage, Thousand Oaks, CA.

Haidt, J. 2001. The emotional dog and its rational tail: A social intuitionist approach to moral judgment. Psychol. Rev. 108:814-834.

Jansen, J., C. D. Steuten, R. J. Renes, N. Aarts, and T. J. Lam. 2010. Debunking the myth of the hard-to-reach farmer: Effective communication on udder health. J. Dairy Sci. 93:1296-1306.

Kauppinen, T., A. Vainio, A. Valros, H. Rita, and K. M. Vesala. 2010. Improving animal welfare: Qualitative and quantitative methodology in the study of farmers' attitudes. Anim. Welf. 19:523-536.

Khan, M. A., D. M. Weary, and M. A. von Keyserlingk. 2011. Invited review: Effects of milk ration on solid feed intake, weaning, and performance in dairy heifers. J. Dairy Sci. 94:1071-1081.

LeBlanc, S. J., K. D. Lissemore, D. F. Kelton, T. F. Duffield, and K. E. Leslie. 2006. Major advances in disease prevention in dairy cattle. J. Dairy Sci. 89:1267-1279.

Maxwell, J. A. 2012. A Realist Approach for Qualitative Research. Sage, Thousand Oaks, CA.

Oreszczyn, S., A. Lane, and S. Carr. 2010. The role of networks of practice and webs of influencers on farmers' engagement with and learning about agricultural innovations. J. Rural Stud. 26:404-417.

Pothmann, H., K. Nechanitzky, F. Sturmlechner, and M. Drillich. 2014. Consultancy to dairy farmers relating to animal health and herd health management on small- and medium-sized farms. J. Dairy Sci. 97:851-860.

Rimal, R. N., and M. K. Lapinski. 2015. A re-explication of social norms, ten years later. Commun. Theory 25:393-409.

Rimal, R. N., and K. Real. 2003. Understanding the influence of perceived norms on behaviors. Commun. Theory 13:184-203.

Saunders, B., J. Sim, T. Kingstone, S. Baker, J. Waterfield, B. Bartlam, H. Burroughs, and C. Jinks. 2018. Saturation in qualitative research: Exploring its conceptualization and operationalization. Qual. Quant. 52:1893-1907.

Sligo, F. X., and C. Massey. 2007. Risk, trust and knowledge networks in farmers' learning. J. Rural Stud. 23:170-182.

Sniehotta, F. F., J. Presseau, and V. Araújo-Soares. 2014. Time to retire the theory of planned behavior. Health Psychol. Rev. 8:1-7.

Southwood, N., and L. Eriksson. 2011. Norms and conventions. Philos. Explor. 14:195-217.

Sumner, C. L., and M. A. G. von Keyserlingk. 2018. Canadian dairy cattle veterinarian perspectives on calf welfare. J. Dairy Sci. 101:10303-10316.

Sumner, C. L., M. A. G. von Keyserlingk, and D. M. Weary. 2018. How benchmarking motivates farmers to improve dairy calf management. J. Dairy Sci. 101:3323-3333.

Swinkels, J. M., A. Hilkens, V. Zoche-Golob, V. Krömker, M. Buddiger, J. Jansen, and T. J. G. M. Lam. 2015. Social influences on the duration of antibiotic treatment of clinical mastitis in dairy cows. J. Dairy Sci. 98:2369-2380.

Triste, L., L. Debryune, J. Vandenabeele, F. Marchand, and L. Lauwers. 2018. Communities of practice for knowledge co-creation on sustainable dairy farming: Features for value creation for farmers. Sustain. Sci. https://doi.org/10.1007/s11625-018-0554-5.

Vaarst, M., S. M. Thamsborg, T. W. Bennedsgaard, H. Houe, C. Enevoldsen, F. M. Aarestrup, and A. de Snoo. 2003. Organic dairy farmers' decision making in the first 2 years after conversion in relation to mastitis treatments. Livest. Prod. Sci. 80:109-120.

Vasseur, E., J. Rushen, A. M. de Passillé, D. Lefebvre, and D. Pellerin. 2010. An advisory tool to improve management practices affecting calf and heifer welfare on dairy farms. J. Dairy Sci. 93:4414-4426.

Wathen, C. N., and H. L. MacMillan. 2018. The role of integrated knowledge translation in intervention research. Prev. Sci. 19:319327. 
Windeyer, M. C., K. E. Leslie, S. M. Godden, D. C. Hodgins, K. D. Lissemore, and S. J. LeBlanc. 2014. Factors associated with morbidity, mortality, and growth of dairy heifer calves up to 3 months of age. Prev. Vet. Med. 113:231-240.

Wolf, C. A., G. T. Tonsor, M. G. S. McKendree, D. U. Thomson, and J. C. Swanson. 2016. Public and farmer perceptions of dairy cattle welfare in the United States. J. Dairy Sci. 99:5892-5903.

\section{ORCIDS}

C. L. Sumner () https://orcid.org/0000-0002-7400-910X

M. A. G. von Keyserlingk @ https://orcid.org/0000-0002-1427-3152

D. M. Weary @ https://orcid.org/0000-0002-0917-3982 